Linde, Dirk; Diebowski, Sandra; Greiner-Petter, Christoph; Schneider, Gerlind:

Evaluation of bone replacement materials in a rabbit cranial defect model using micro CT and hard tissue histology

Zuerst erschienen in: $\quad$ Biomedical Engineering = Biomedizinische Technik. - Berlin [u.a.] : de Gruyter. - 57 (2012), Suppl. 1, Track-D, p. 557-560.

Erstveröffentlichung: 2012-08-27

ISSN (online): $\quad$ 1862-278X

ISSN (print): $\quad$ 0013-5585

DOI: $\quad 10.1515 / \mathrm{bmt}-2012-4307$

[Zuletzt gesehen: 2019-08-12]

„Im Rahmen der hochschulweiten Open-Access-Strategie für die Zweitveröffentlichung identifiziert durch die Universitätsbibliothek IImenau."

"Within the academic Open Access Strategy identified for deposition by IImenau University Library."

„Dieser Beitrag ist mit Zustimmung des Rechteinhabers aufgrund einer (DFGgeförderten) Allianz- bzw. Nationallizenz frei zugänglich.“

"This publication is with permission of the rights owner freely accessible due to an Alliance licence and a national licence (funded by the DFG, German

DFG

Research Foundation) respectively." 


\title{
Evaluation of bone replacement materials in a rabbit cranial defect model using micro CT and hard tissue histology
}

\author{
D. Linde ${ }^{1}$, S. Diebowski ${ }^{1}$, C. Greiner-Petter ${ }^{2}$, G. Schneider ${ }^{1}$ \\ ${ }^{1}$ Friedrich Schiller University Hospital, Department of Othorhinolaryngology, Jena, Germany, \\ ${ }^{2}$ Ilmenau University of Technology, Department of Mechatronics, Ilmenau, Germany \\ dirk.linde@med.uni-jena.de
}

\begin{abstract}
Introduction: For functional and structural restoration of bone deficiencies, various resorbable and nonresorbable alloplastic materials have been introduced, including metals, polymers and ceramics. However, an "optimal" artificial replacement for craniofacial bone has not been found yet and the search for improved reconstruction methods and alternative materials is going on. To assess and compare biocompatibility and osseointegration of these materials, adequate animal test models are indispensable.
\end{abstract}

Methods: In a rabbit cranial defect model, biocompatibility and osseointegration of polymeric and composite bone replacement materials were evaluated at different time points after implantation. Calvaria including implants and surrounding tissue were explanted and embedded in methacrylate resin. The samples were scanned with a nanotom ${ }^{\circledR}$ (phoenix|x-ray) $\mu \mathrm{CT}$ scanner and proceeded for histological examination by sawing-grinding technique. Avizo ${ }^{\circledR}$ Fire (vsg) software was used for visualisation and processing of $\mu \mathrm{CT}$ data. Qualitative and morphometric evaluation of osseointegration and fibrous encapsulation was performed on undecalcified histologic preparations of the explants, and on 3D reconstructions plus virtual slices derived from corresponding $3 \mathrm{D} \mu \mathrm{CT}$ datasets.

Results: The obtained 3D $\mu \mathrm{CT}$ data enabled a comprehensive qualitative and quantitative assessment of osseointegration and biodegradation of radioopaque composite implants. Prerequisite for visualization and discrimination of materials by $\mu \mathrm{CT}$ is a significant difference of their hounsfield values. Due to this limitation, radiolucent polymeric implant materials and soft tissue could not be distinguished from embedding resin. In contrast, histologic preparations of undecalcified hard tissue and implant materials enabled detailed visualization and examination of all tissues and implant materials. The substantial disadvantage of hard tissue histology was the inevitable loss of information due to small number of slices and large gaps between specimens yielded by this method.

Conclusion: To obtain comprehensive and quantifiable information about biodegradation, biocompatibility and osseointegration of alloplastic bone replacement materials, $\mu \mathrm{CT}$ scans as well as histologic evaluation should be performed.

\section{Introduction}

For functional and structural restoration of bone deficiencies in the skull, various resorbable and nonresorbable alloplastic materials have been introduced, including metals, polymers, ceramics and composites (Sanan and Haines 1997). However, an "optimal" artificial replacement for craniofacial bone has not been found yet and the search for improved reconstruction methods and alternative materials is going on (Engstrand 2012).

To assess and compare biocompatibility and osseointegration of these materials, adequate animal test models are indispensable. To answer these needs, in Jena University Hospital an animal model had been developed and established by the Biomaterials Lab of the Department of Othorhinolaryngology. The "Jenaer Schädelmodell" (Jena Skull Model), intended for evaluation of bone replacement materials, is used in particular for applications in calvarial parts of the skull. It provides significant information about osseointegration of implants, biodegradation, foreign body response and effects on nervous tissue (Schneider et al. 2010, Voigt and Schneider 2009).

In the ongoing efforts to obtain an optimum of significant information by means of this animal model, computer assisted quantitative analyses and modern imaging methods like high resolution computed tomography $(\mu \mathrm{CT})$ (Robinson et al. 2005, Ritman 2004, Ritman 2011) have the potential to become essential complements to well established "classic" methods like histologic analyses of undecalcified hard tissue (Donath and Breuner 1982).

\section{Methods}

Biocompatibility and osseointegration of a composite bone replacement material were evaluated in a rabbit skull model for calvarial defects (Schneider et al. 2010, Voigt and Schneider 2009). 
Each animal received two composite implants and two implants consisting of the composite components, microporous tricalcium phosphate (TCP) and methacrylate polymer.

With permission from Thüringer Landesamt für Lebensmittelsicherheit und Verbraucherschutz (Reg.Nr.: 02036/08), a total of 18 female New Zealand White rabbits were used in this study. For implantations, anesthetized animals had undergone trepanations, resulting in four 5 $\mathrm{mm}$ diameter calvarial defects per animal. After removing he dura underneath the trepanations, cylindrical implants were fitted into the defects. Implants were fixed by sutures closing periost and skin above the implantation sites.

Three, six and nine months after implantation, respectively, animals were sacrificed and the calvaria including implants and surrounding tissue were explanted. Explants were embedded in Technovit 7200 VLC methacrylate resin (Heraeus-Kulzer, Germany). For histological examination, the undecalcified explants were proceeded by sawinggrinding technique and histological staining (Donath and Breuner 1982).

Prior to histological proceeding, resin embedded specimen explanted after nine months implantation time were scanned with a nanotom ${ }^{\circledR} \mu \mathrm{CT}$ scanner (phoenix|x-ray, GE-Sensing \& Inspection Technologies GmbH, Germany) at a resolution of $14 \mu \mathrm{m}$ per voxel. For visualisation and processing of $3 \mathrm{D} \mu \mathrm{CT}$ data, Avizo ${ }^{\circledR}$-Fire $3 \mathrm{D}$ visualisation software (vsg - Visualization Sciences Group, France) was used.

Microscopic images of undecalcified histologic preparations and $3 \mathrm{D}$ reconstructions as well as series of virtual slices derived from corresponding $\mu \mathrm{CT}$ datasets were used for qualitative and quantitative evaluation of osseointegration and fibrous encapsulation.

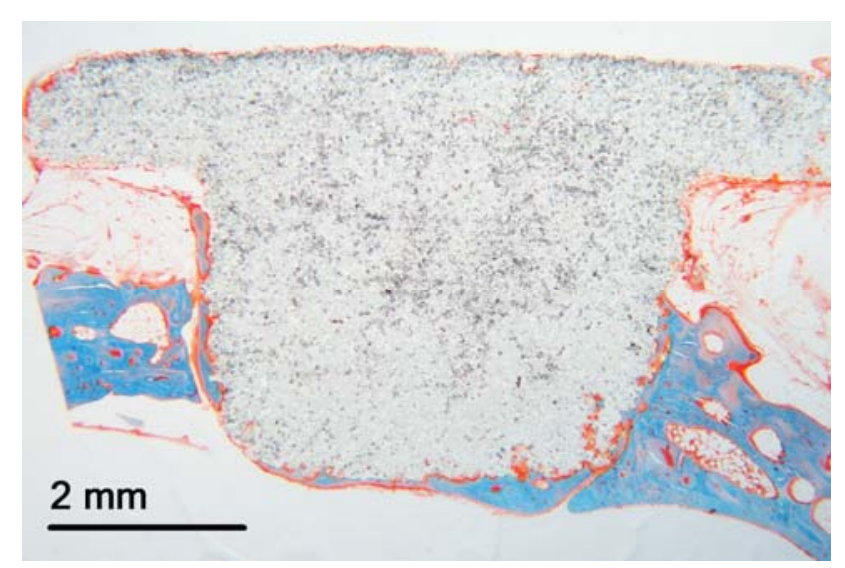

Image 1 Microphotograph of composite implant, soft tissue (red) and bone (blue); mod.Masson-Goldner staining

\section{Results}

In the present animal study, all implanted materials were well tolerated by the surrounding tissue showing good biocompatibility and osseointegration. In contrast to the composite and polymer implants, the microporous TCP implants had undergone significant biodegradation which was not complete yet after an implantation period of 9 months. Composite and polymer implants were showing fibrous encapsulation, which was diminishing over the tested time period, enabling an increasing ratio of direct bone-to-implant contact (BIC). In direct comparison to pure polymer, composite implants showed thinner layers of fibroconnective tissue at the bone-implant interface indicating lower intensity of foreign body response, and gradually enhanced osseointegration with higher BIC ratios.

The evaluation approaches used in this study, hard tissue histology (image 1) and $\mu \mathrm{CT}$ scans (image 2), enabled to assess biocompatibility, osseointegration and biodegradation of implant materials. Both approaches have specific advantages and limitations and were in part complementary in evaluation of the explanted specimens.

Prerequisite for the non-destructive visualization and discrimination of materials by $\mu \mathrm{CT}$ are significant differences of their hounsfield values. Due to this limitation, radiolucent polymeric implant materials and soft tissue could not be distinguished from embedding resin.

On the other hand, three-dimensional $\mu \mathrm{CT}$ data enabled a comprehensive qualitative and quantitative threedimensional assessment of osseointegration and biodegradation of radioopaque materials, i.e. TCP and TCP-polmer composites (image 2). Composite implants are shown in image $2 \mathrm{a}$ in upper right and lower left trepanation defects. Polymer implants, having the same radiodensity as the embedding resin, could not be visualised by $\mu \mathrm{CT}$ (image 2a, lower right trepanation slot). A virtual slice in image $2 \mathrm{~b}$ shows on the left a composite implant and on the right the slot of a polymer implant (appearing empty). An example of biodegrading microporous TCP implant is visualised in the upper left trepanation defect in image $2 \mathrm{a}$. A virtual slice is shown in image $2 \mathrm{c}$ (left side: TCP; right side: composite).

In contrary to these limitations of $\mu \mathrm{CT}$ data, histologic preparation of undecalcified hard tissue and implant materials enabled detailed visualization of all implant materials at a microscopic resolution. In addition, embedded cells and extracellular matrix were visualised by histologic staining, providing detailed information about cellular inflammation reactions and layers of fibroconnective tissue indicating intensity of foreign body responses. The histological findings obtained by sawing-grinding technique were essential to assess biocompatibility of the implanted materials. An example of a composite implant surrounded by minor amounts of fibrous encapsulation layers (stained red) and being partially in direct contact with bone (stained blue) is shown in image 1 . 
Unlike the $\mu \mathrm{CT}$-scans, the sawing-grinding technique for microscopic preparations of undecalcified hard tissue is a destructive method. A second fundamental disadvantage and inherent limitation of this preparation method is the inevitable loss of information due to large gaps (approx. $500 \mu \mathrm{m})$ between histologic sections, resulting in limited number of sections yielded by this method.
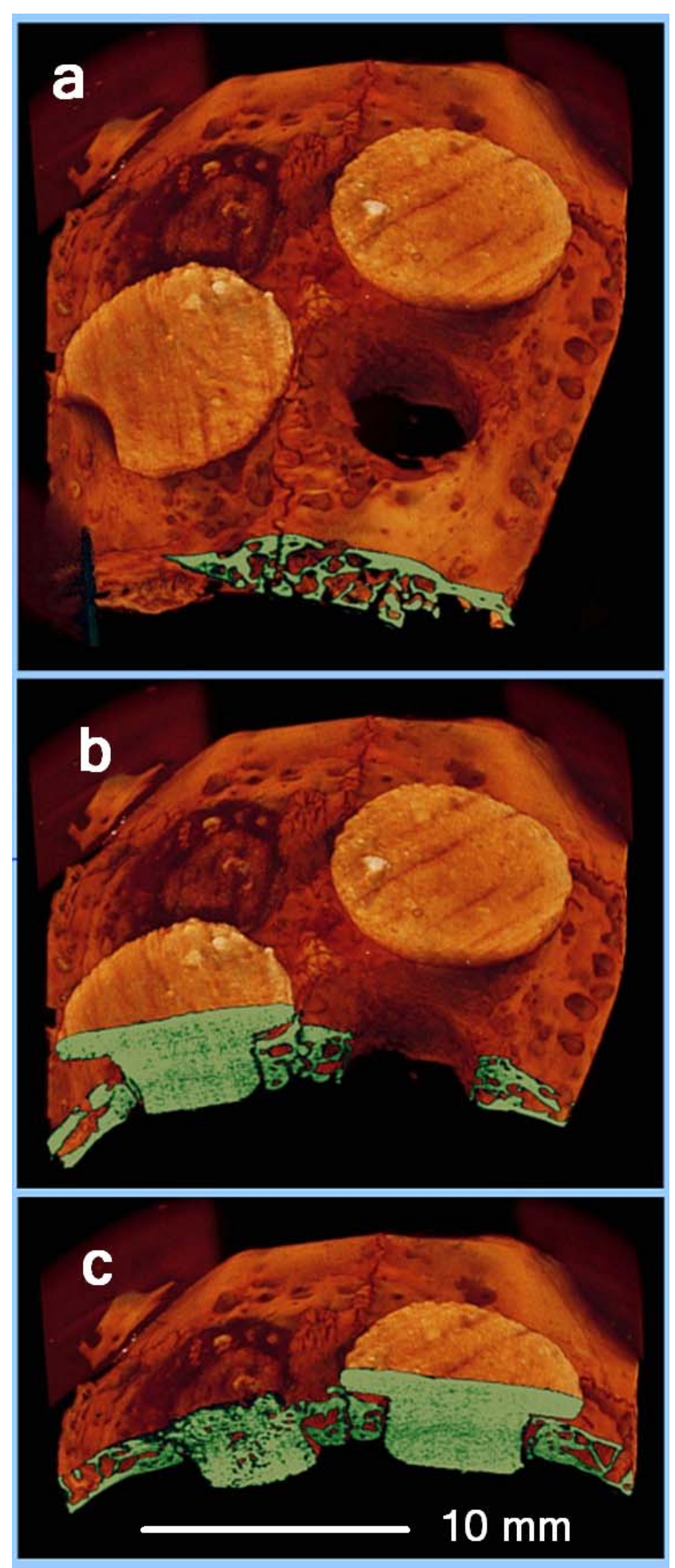

Image 2 a,b,c $\mu \mathrm{CT}$ scan of explanted rabbit calvaria with implants in trepanation defects; surface and virtual slices
Like in other animal models for evaluation of implants in contact to bone (Draenert et al.), it turned out to be advantageous to gain complementary information by an adequate three-dimensional imaging technique. $\mu \mathrm{CT}$ scans were evaluated as being suitable, providing comprehensive three-dimensional information about bone apposition to implant surface and about topographical orientation of histologic sections gained by sawing-grinding technique.

Prerequisite for the use of $\mu \mathrm{CT}$ scans in three-dimensional evaluation of osseointegration is the distinction of implants from bone by segmentation of the $\mu \mathrm{CT}$ dataset volume. If the scanned materials have significantly different radiodensities, discrimination can be performed efficiently by the automatic or semi-automatic segmentation algorithms provided by $3 \mathrm{D}$ visualisation applications. This approach is for example applicable to distinct bone from metallic implant materials like titanium alloys (Sarve et al. 2011).

Composite implants evaluated in the present study and the adjacent bone turned out to have very similar radiodensities. Under this precondition, distinction of implant and bone by three-dimensional segmentation would be difficult and extremely laborious, rendering a three-dimensional evaluation via segmentation of 3D data very inefficient.

For this reason, alternative methods were employed to gain detailed information about osseointegration of composite implants from $\mu \mathrm{CT}$ datasets. To generate defined series of virtual slices from 3D datasets, an algorithm was devised and implemented in Avizo ${ }^{\circledR}$-Fire 3D visualisation software. Single virtual slices as well as comprehensive sets of virtual slices were generated from $\mu \mathrm{CT}$ scans and further evaluated in parallel with images of the corresponding histologic preparations.

In addition to qualitative evaluations, an important objective was to establish methods for morphometric analysis of both microscopic images gained via hard tissue histology and virtual slices derived from $\mu \mathrm{CT}$ scans.

A dedicated software application was developed to enable an efficient quantitative evaluation of explanted specimens of the in-vivo model. The software supports structured and ergonomic input of data concerning experimental setup. It enables accurate and detailed measurement of both boneimplant distances and thickness of fibrous tissue layers in the gaps between bone and implants. Output data are stored in a standardised format to enable consistent processing with statistics software and database applications.

\section{Conclusion}

In animal implantation models like the "Jenaer Schädelmodell", it is recommended to perform $\mu \mathrm{CT}$ scans as well as histologic evaluations to obtain comprehensive and quantifiable information about biodegradation, biocompatibility and osseointegration of alloplastic bone replacement materials. 


\section{$5 \quad$ References}

Donath K, Breuner G. 1982. A method for the study of undecalcified bones and teeth with attached soft tissues. The Säge-Schliff (sawing and grinding) technique. J Oral Pathol, 11 (4):318-326.

Draenert ME, Draenert AI, Forriol F, Erler M, Kunzelmann KH, Hickel R, Draenert K. 2012. Value and limits of mu-CT for nondemineralized bone tissue processing. Microscopy Research and Technique, 75 (4):416-424.

Engstrand T. 2012. Biomaterials and Biologics in Craniofacial Reconstruction. Journal of Craniofacial Surgery, 23 (1):239-242.

Ritman EL. 2004. Micro-computed tomography-current status and developments. Annual Review of Biomedical Engineering, 6:185-208.

Ritman EL. 2011. Current status of developments and applications of micro-CT. Annual Review of Biomedical Engineering, 13:531-552.

Robinson S, Suomalainen A, Kortesniemi M. 2005. micro-CT. European Journal of Radiology, 56 (2):185-191.

Sanan A, Haines SJ. 1997. Repairing holes in the head: A history of cranioplasty. Neurosurgery, 40 (3):588-603.

Sarve H, Lindblad J, Borgefors G, Johansson CB. 2011. Extracting 3D information on bone remodeling in the proximity of titanium implants in SR mu CT image volumes. Computer Methods and Programs in Biomedicine, 102 (1):25-34.

Schneider G, Blechschmidt K, Linde D, Litschko P, Körbs T, Beleites E. 2010. Bone regeneration with glass ceramic implants and calcium phosphate cements in a rabbit cranial defect model. J Mater Sci Mater Med, 21 (10):2853-2859.

Voigt S, Schneider G. 2009. Das Jenaer Schädelmodell. Tübingen: Jahrestagung der DGBM. 